HIGHLIGHTS OF PRESCRIBING INFORMATION

These highlights do not include all the information needed to use CABLIVI ${ }^{\circledR}$ safely and effectively. See full prescribing information for CABLIVI.

CABLIVI (caplacizumab-yhdp) for injection, for intravenous or subcutaneous use

Initial U.S. Approval: 2019

-INDICATIONS AND USAGE-

CABLIVI is a von Willebrand factor (vWF)-directed antibody fragment indicated for the treatment of adult patients with acquired thrombotic thrombocytopenic purpura (aTTP), in combination with plasma exchange and immunosuppressive therapy. (1)

\section{DOSAGE AND ADMINISTRATION}

- CABLIVI should be administered upon the initiation of plasma exchange therapy. The recommended dose of CABLIVI is as follows: (2.1)

o First day of treatment: $11 \mathrm{mg}$ bolus intravenous injection at least 15 minutes prior to plasma exchange followed by an $11 \mathrm{mg}$ subcutaneous injection after completion of plasma exchange on day 1 .

o Subsequent treatment during daily plasma exchange: $11 \mathrm{mg}$ subcutaneous injection once daily following plasma exchange.

o Treatment after the plasma exchange period: $11 \mathrm{mg}$ subcutaneous injection once daily for 30 days beyond the last plasma exchange.

o If after initial treatment course, sign(s) of persistent underlying disease such as suppressed ADAMTS13 activity levels remain present, treatment may be extended for a maximum of 28 days.

o Discontinue CABLIVI if the patient experiences more than 2 recurrences of aTTP, while on CABLIVI. intravenous injection. Administer subsequent doses subcutaneously in the abdomen. (2.3)

-DOSAGE FORMS AND STRENGTHS

For injection: $11 \mathrm{mg}$ as a lyophilized powder in a single-dose vial. (3) CONTRAINDICATIONS-

Previous severe hypersensitivity reaction to caplacizumab-yhdp or any of the excipients. (4)

\section{WARNINGS AND PRECAUTIONS}

Bleeding: Severe bleeding can occur; risk is increased in patients with underlying coagulopathies. If clinically significant bleeding occurs, interrupt treatment. Withhold CABLIVI 7 days prior to elective surgery, dental procedures, or other invasive interventions. (5.1)

\section{ADVERSE REACTIONS}

Most common adverse reactions (incidence $>15 \%$ ) are epistaxis, headache, and gingival bleeding. (6.1)

To report SUSPECTED ADVERSE REACTIONS, contact Ablynx US at 1-800-745-4447 or FDA at 1-800-FDA-1088 or www.fda.gov/medwatch.

\section{DRUG INTERACTIONS}

Concomitant use of anticoagulants with CABLIVI may increase the risk of bleeding. Monitor closely for bleeding with concomitant use. (7)

See 17 for PATIENT COUNSELING INFORMATION and FDAapproved patient labeling.

Revised: 02/2019

- The first dose should be administered by a healthcare provider as a bolus

\author{
FULL PRESCRIBING INFORMATION: CONTENTS* \\ 1 INDICATIONS AND USAGE \\ 2 DOSAGE AND ADMINISTRATION \\ 2.1 Recommended Dose and Schedules \\ 2.2 Discontinuation for Surgery and Other Interventions \\ 2.3 Reconstitution and Administration Instructions \\ 3 DOSAGE FORMS AND STRENGTHS \\ 4 CONTRAINDICATIONS \\ 5 WARNINGS AND PRECAUTIONS \\ 5.1 Bleeding \\ 6 ADVERSE REACTIONS \\ 6.1 Clinical Trials Experience \\ 6.2 Immunogenicity \\ 7 DRUG INTERACTIONS \\ 8 USE IN SPECIFIC POPULATIONS \\ 8.1 Pregnancy \\ 8.2 Lactation \\ 8.4 Pediatric Use
}

\author{
8.5 Geriatric Use \\ 8.6 Hepatic Impairment \\ 10 OVERDOSAGE \\ 11 DESCRIPTION \\ 12 CLINICAL PHARMACOLOGY \\ 12.1 Mechanism of Action \\ 12.2 Pharmacodynamics \\ 12.3 Pharmacokinetics \\ 13 NONCLINICAL TOXICOLOGY \\ 13.1 Carcinogenesis, Mutagenesis, Impairment of Fertility \\ 14 CLINICAL STUDIES \\ 16 HOW SUPPLIED/STORAGE AND HANDLING \\ 16.1 How Supplied \\ 16.2 Storage \\ 17 PATIENT COUNSELING INFORMATION
}

*Sections or subsections omitted from the full prescribing information are not listed. 


\section{FULL PRESCRIBING INFORMATION}

\section{INDICATIONS AND USAGE}

CABLIVI is indicated for the treatment of adult patients with acquired thrombotic thrombocytopenic purpura (aTTP), in combination with plasma exchange and immunosuppressive therapy.

\section{DOSAGE AND ADMINISTRATION}

\subsection{Recommended Dose and Schedules}

CABLIVI should be administered upon initiation of plasma exchange therapy. The recommended dose of CABLIVI is as follows:

- First day of treatment: $11 \mathrm{mg}$ bolus intravenous injection at least 15 minutes prior to plasma exchange followed by an $11 \mathrm{mg}$ subcutaneous injection after completion of plasma exchange on day 1.

- $\quad$ Subsequent days of treatment during daily plasma exchange: $11 \mathrm{mg}$ subcutaneous injection once daily following plasma exchange.

- Treatment after plasma exchange period: $11 \mathrm{mg}$ subcutaneous injection once daily continuing for 30 days following the last daily plasma exchange. If after initial treatment course, sign(s) of persistent underlying disease such as suppressed ADAMTS13 activity levels remain present, treatment may be extended for a maximum of 28 days.

Discontinue CABLIVI if the patient experiences more than 2 recurrences of aTTP, while on CABLIVI.

\section{Missed Dose}

If a dose of CABLIVI is missed during the plasma exchange period, it should be given as soon as possible. If a dose of CABLIVI is missed after the plasma exchange period, it can be administered within 12 hours of the scheduled time of administration. Beyond 12 hours, the missed dose should be skipped and the next daily dose administered according to the usual dosing schedule.

\subsection{Discontinuation for Surgery and Other Interventions}

Withhold CABLIVI treatment 7 days prior to elective surgery, dental procedures, or other invasive interventions [see Warnings and Precautions (5.1)].

\subsection{Reconstitution and Administration Instructions}

The first dose of CABLIVI should be administered by a healthcare provider as a bolus intravenous injection. Administer subsequent doses subcutaneously in the abdomen. Avoid injections around the navel. Do not administer consecutive injections in the same abdominal quadrant.

Patients or caregivers may inject CABLIVI subcutaneously after proper training on the preparation and administration of CABLIVI, including aseptic technique [see Instructions for 
Use].

- Ensure the CABLIVI vial and diluent syringe are at room temperature.

- Reconstitute CABLIVI before administration using the provided syringe containing $1 \mathrm{~mL}$ Sterile Water for Injection, USP, to yield an $11 \mathrm{mg} / \mathrm{mL}$ single-dose solution.

- Using aseptic technique throughout the preparation of the solution, attach the vial adapter to the vial containing CABLIVI.

- Remove the plastic cap from the syringe and attach it to the vial adapter by twisting it clockwise until it cannot twist any further.

- Slowly push the syringe plunger down until the syringe is empty. Do not remove the syringe from the vial adapter.

- Gently swirl the vial until the cake or powder is completely dissolved. Do not shake.

- Visually inspect that the reconstituted solution is clear and colorless.

- Withdraw all of the clear, colorless reconstituted solution from the vial into the syringe. Label the CABLIVI syringe.

- Administer the full amount of reconstituted solution.

- For the initial intravenous injection, if using an intravenous line, the glass syringe should be connected to a standard Luer lock (and not a needleless connector) and flushed with either $0.9 \%$ Sodium Chloride Injection, USP, or 5\% Dextrose Injection, USP.

- Use the CABLIVI solution immediately. If not, use CABLIVI within 4 hours after reconstitution when stored in the refrigerator at $2^{\circ} \mathrm{C}$ to $8^{\circ} \mathrm{C}\left(36^{\circ} \mathrm{F}\right.$ to $\left.46^{\circ} \mathrm{F}\right)$.

\section{DOSAGE FORMS AND STRENGTHS}

For injection: $11 \mathrm{mg}$ as a white lyophilized powder in a single-dose vial.

\section{CONTRAINDICATIONS}

CABLIVI is contraindicated in patients with a previous severe hypersensitivity reaction to caplacizumab-yhdp or to any of the excipients. Hypersensitivity reactions have included urticaria [see Adverse Reactions (6.1)].

\section{WARNINGS AND PRECAUTIONS}

\section{$5.1 \quad$ Bleeding}

CABLIVI increases the risk of bleeding [see Adverse Reactions (6.1)]. In clinical studies, severe bleeding adverse reactions of epistaxis, gingival bleeding, upper gastrointestinal hemorrhage, and metrorrhagia were each reported in $1 \%$ of subjects. Overall, bleeding events occurred in approximately $58 \%$ of patients on CABLIVI versus $43 \%$ of patients on placebo.

The risk of bleeding is increased in patients with underlying coagulopathies (e.g. hemophilia, other coagulation factor deficiencies). It is also increased with concomitant use of CABLIVI with drugs affecting hemostasis and coagulation [see Drug Interactions (7) and Clinical Pharmacology (12.3)]. 
Interrupt use of CABLIVI if clinically significant bleeding occurs. If needed, von Willebrand factor concentrate may be administered to rapidly correct hemostasis. If CABLIVI is restarted, monitor closely for signs of bleeding.

Withhold CABLIVI for 7 days prior to elective surgery, dental procedures or other invasive interventions. If emergency surgery is needed, the use of von Willebrand factor concentrate may be considered to correct hemostasis. After the risk of surgical bleeding has resolved, and CABLIVI is resumed, monitor closely for signs of bleeding.

\section{ADVERSE REACTIONS}

The following clinically significant adverse reactions are also discussed in other sections of the labeling:

- Bleeding [see Warnings and Precautions (5.1)]

\subsection{Clinical Trials Experience}

Because clinical trials are conducted under widely varying conditions, adverse reaction rates observed in the clinical trials of a drug cannot be directly compared to rates in the clinical trials of another drug and may not reflect the rates observed in practice.

The safety of CABLIVI was evaluated in two placebo-controlled clinical studies (HERCULES, in which 71 patients received CABLIVI; and TITAN, in which 35 patients received CABLIVI). The data described below and in the Warnings and Precautions reflect exposure to CABLIVI during the blinded periods of both studies, which include 106 patients with aTTP who received at least one dose, age 18 to 79 years, of whom $69 \%$ were female and $73 \%$ were White. The median treatment duration with CABLIVI was 35 days (range 1-77 days).

The most frequently reported adverse reactions $(>15 \%)$ were epistaxis, headache and gingival bleeding. Seven patients (7\%) in the CABLIVI group experienced an adverse reaction leading to study drug discontinuation. None of the adverse reactions leading to discontinuation were observed in more than $1 \%$ of patients.

Among 106 patients treated with CABLIVI during the TITAN and HERCULES studies, serious bleeding adverse reactions reported in $\geq 2 \%$ patients included epistaxis (4\%) and subarachnoid hemorrhage $(2 \%)$.

Adverse reactions that occurred in $\geq 2 \%$ of patients treated with CABLIVI and more frequently than in those treated with placebo across the pooled data from the two trials are summarized in Table 1. Urticaria was seen during plasma exchange.

Table 1: Adverse Reactions in $\geq 2 \%$ of Patients Treated with CABLIVI and More Frequent than Placebo during the Blinded Periods of aTTP Studies (HERCULES and TITAN)

\begin{tabular}{|l|c|c|}
\hline Adverse Reaction by Body System & $\begin{array}{c}\text { CABLIVI } \\
(\mathrm{N}=106) \\
\mathrm{n}(\%)\end{array}$ & $\begin{array}{c}\text { Placebo } \\
(\mathrm{N}=110) \\
\mathrm{n}(\%)\end{array}$ \\
\hline Gastrointestinal disorders & $17(16)$ & $3(3)$ \\
\hline Gingival bleeding & $4(4)$ & $0(0)$ \\
\hline Rectal hemorrhage & $3(3)$ & $1(1)$ \\
\hline Abdominal wall hematoma &
\end{tabular}




\begin{tabular}{|c|c|c|}
\hline Adverse Reaction by Body System & $\begin{array}{c}\text { CABLIVI } \\
(\mathrm{N}=106) \\
\mathrm{n}(\%)\end{array}$ & $\begin{array}{c}\text { Placebo } \\
(\mathrm{N}=110) \\
\mathrm{n}(\%)\end{array}$ \\
\hline \multicolumn{3}{|c|}{ General disorders and administration site conditions } \\
\hline Fatigue & $16(15)$ & $10(9)$ \\
\hline Pyrexia & $14(13)$ & $12(11)$ \\
\hline Injection site hemorrhage & $6(6)$ & $1(1)$ \\
\hline Catheter site hemorrhage & $6(6)$ & $5(5)$ \\
\hline Injection site pruritus & $3(3)$ & $0(0)$ \\
\hline \multicolumn{3}{|c|}{ Musculoskeletal and connective tissue disorders } \\
\hline Back pain & $7(7)$ & $4(4)$ \\
\hline Myalgia & $6(6)$ & $2(2)$ \\
\hline \multicolumn{3}{|l|}{ Nervous system disorders } \\
\hline Headache & $22(21)$ & $15(14)$ \\
\hline Paresthesia & $13(12)$ & $11(10)$ \\
\hline \multicolumn{3}{|l|}{ Renal and urinary disorders } \\
\hline Urinary tract infection & $6(6)$ & $4(4)$ \\
\hline Hematuria & $4(4)$ & $3(3)$ \\
\hline \multicolumn{3}{|c|}{ Reproductive system and breast disorders } \\
\hline Vaginal hemorrhage & $5(5)$ & $2(2)$ \\
\hline Menorrhagia & $4(4)$ & $1(1)$ \\
\hline \multicolumn{3}{|c|}{ Respiratory, thoracic and mediastinal disorders } \\
\hline Epistaxis & $31(29)$ & $6(6)$ \\
\hline Dyspnea & $10(9)$ & $5(5)$ \\
\hline \multicolumn{3}{|c|}{ Skin and subcutaneous tissue disorders } \\
\hline Urticaria & $15(14)$ & $7(6)$ \\
\hline
\end{tabular}

\subsection{Immunogenicity}

As with all therapeutic proteins, there is potential for immunogenicity. The detection of antibody formation is highly dependent on the sensitivity and specificity of the assay. Additionally, the observed incidence of antibody (including neutralizing antibody) positivity in an assay may be influenced by several factors including assay methodology, sample handling, timing of sample collection, concomitant medications, and underlying disease. For these reasons, comparison of the incidence of antibodies to caplacizumab-yhdp in the studies described below, with the incidence of antibodies in other studies, or to other products, may be misleading.

The prevalence of pre-existing antibodies binding to caplacizumab-yhdp observed during clinical studies and during evaluation of commercially available human samples varied between $4 \%$ and $63 \%$. In aTTP patients, pre-existing antibodies can be produced by the patient or can originate from donor plasma during plasma exchange. No clinically apparent impact of these pre-existing antibodies on clinical efficacy or safety was found. Treatment-emergent anti-drug antibodies (TE ADA) against caplacizumab-yhdp were detected in 3\% of patients treated with CABLIVI in the HERCULES study. In the HERCULES study, TE ADA were further characterized as having neutralizing potential. There was no clinically apparent impact on clinical efficacy or safety [see Clinical Pharmacology (12.3)]. 


\section{DRUG INTERACTIONS}

\section{Concomitant Use of Anticoagulants}

Concomitant use of CABLIVI with any anticoagulant may increase the risk of bleeding. Assess and monitor closely for bleeding with concomitant use [see Warnings and Precautions (5.1) and Clinical Pharmacology (12.3)].

\section{USE IN SPECIFIC POPULATIONS}

\subsection{Pregnancy}

\section{Risk Summary}

There are no available data on CABLIVI use in pregnant women to inform a drug-associated risk of major birth defects and miscarriage. However, there are potential risks of hemorrhage in the mother and fetus associated with use of CABLIVI (see Clinical Considerations). In animal reproduction studies, there was no evidence of adverse developmental outcomes with intramuscular administration of caplacizumab-yhdp during organogenesis in guinea pigs at exposures approximately 30 times the AUC in humans at the recommended subcutaneous injection dose of $11 \mathrm{mg}$ (see Data).

All pregnancies have a background risk of birth defect, loss, or other adverse outcomes. The background rate of major birth defects and miscarriage in the indicated population is unknown. In the U.S. general population, the estimated background rate of major birth defects and miscarriage in clinically recognized pregnancies is $2 \%$ to $4 \%$ and $15 \%$ to $20 \%$, respectively.

\section{Clinical Considerations}

\section{Fetal/neonatal adverse reactions}

CABLIVI may increase the risk of bleeding in the fetus and neonate. Monitor neonates for bleeding [see Warnings and Precautions (5.1)].

\section{Maternal adverse reactions}

All patients receiving CABLIVI, including pregnant women, are at risk for bleeding. Pregnant women receiving CABLIVI should be carefully monitored for evidence of excessive bleeding [see Warnings and Precautions (5.1)].

\section{Data}

\section{Animal data}

Two separate reproduction studies were conducted in pregnant guinea pigs with administration of caplacizumab-yhdp during the organogenesis period.

In an embryo-fetal development study, caplacizumab-yhdp was administered intramuscularly at doses up to $20 \mathrm{mg} / \mathrm{kg} /$ day from gestational day (GD) 6 to GD 41 in guinea pigs. No maternal toxicity or adverse developmental outcomes were observed.

In a toxicokinetic study assessing the exposure of caplacizumab-yhdp in the dams and fetuses, caplacizumab-yhdp was administered once daily to female guinea pigs at doses up to $40 \mathrm{mg} / \mathrm{kg} /$ day (corresponding to a drug exposure of approximately 30 times the AUC in humans at the recommended dose of $11 \mathrm{mg}$ ) by intramuscular injection from GD 6 to GD 41 or GD 61 . Exposure to caplacizumab-yhdp was observed in the dams and fetuses, with no effects on 
embryo-fetal development.

\subsection{Lactation}

\section{Risk Summary}

There is no information regarding the presence of caplacizumab-yhdp in human milk, the effects on the breastfed child or the effects on milk production.

The developmental and health benefits of breastfeeding should be considered along with the mother's clinical need for CABLIVI and any potential adverse effects on the breastfed child from CABLIVI, or from the underlying maternal condition.

\subsection{Pediatric Use}

The safety and effectiveness of CABLIVI in pediatric patients have not been established.

\subsection{Geriatric Use}

Clinical studies of CABLIVI did not include sufficient numbers of subjects aged 65 and over to determine whether they respond differently from younger subjects.

\subsection{Hepatic Impairment}

No formal studies with CABLIVI have been conducted in patients with severe acute or chronic hepatic impairment and no data regarding the use of CABLIVI in these populations are available. Due to a potential increased risk of bleeding, use of CABLIVI in patients with severe hepatic impairment requires close monitoring for bleeding [see Warnings and Precautions (5.1)].

\section{OVERDOSAGE}

In case of overdose, based on the pharmacological action of CABLIVI, there is the potential for an increased risk of bleeding [see Warnings and Precautions (5.1)]. Close monitoring for signs and symptoms of bleeding is recommended. If needed, the use of von Willebrand factor concentrate could be considered to correct hemostasis.

\section{DESCRIPTION}

Caplacizumab-yhdp is a von Willebrand factor (vWF)-directed antibody fragment that consists of two identical humanized building blocks, linked by a three-alanine linker. Caplacizumab-yhdp is produced in Escherichia coli by recombinant DNA technology and has an approximate molecular weight of $28 \mathrm{kDa}$.

CABLIVI (caplacizumab-yhdp) for injection is a sterile, white, preservative-free, lyophilized powder. Each single-dose vial delivers $11 \mathrm{mg}$ caplacizumab-yhdp, anhydrous citric acid $(0.18 \mathrm{mg})$, polysorbate- $80(0.10 \mathrm{mg})$, sucrose $(62 \mathrm{mg})$, and trisodium citrate dihydrate $(4.91 \mathrm{mg})$. After reconstitution with $1 \mathrm{~mL}$ of Sterile Water for Injection, USP, the final concentration is $11 \mathrm{mg} / \mathrm{mL}$, at a $\mathrm{pH}$ of approximately 6.5 .

\section{CLINICAL PHARMACOLOGY}

\subsection{Mechanism of Action}

Caplacizumab-yhdp targets the A1-domain of vWF, and inhibits the interaction between vWF 
and platelets, thereby reducing both vWF-mediated platelet adhesion and platelet consumption.

\subsection{Pharmacodynamics}

Ristocetin cofactor (RICO) activity was used to assess vWF activity. Subcutaneous doses of caplacizumab-yhdp at greater than or equal to the approved recommended dosage to healthy subjects and patients with aTTP decreased RICO activity levels to below 20\% approximately 4 hours post-dose. RICO activity returned to baseline values within 7 days of drug discontinuation.

Caplacizumab-yhdp decreased vWF antigen and factor VIII:C levels. These reductions were transient and returned to baseline upon cessation of treatment.

\subsection{Pharmacokinetics}

Caplacizumab-yhdp pharmacokinetics depends on the expression of the target vWF and are not dose proportional. Higher levels of vWF antigen increase the fraction of drug-target complex retained in the circulation. Steady-state was reached following the first administration of CABLIVI in healthy subjects, with minimal accumulation. Following a single subcutaneous dose of $10 \mathrm{mg}$ caplacizumab-yhdp to healthy subjects the mean $(\mathrm{CV} \%)$ peak concentration $\left(\mathrm{C}_{\max }\right)$ was $528(20 \%) \mathrm{ng} / \mathrm{ml}$ and $\mathrm{AUC}_{0-24}$ was 7951 (16\%). Following subcutaneous dosing of $10 \mathrm{mg}$ caplacizumab-yhdp daily for 14 days to healthy subjects, the mean $(\mathrm{CV} \%) \mathrm{C}_{\max }$ was $348(30 \%)$ $\mathrm{ng} / \mathrm{ml}$ and $\mathrm{AUC}_{0-\tau}$ was $6808(26 \%) \mathrm{hr} \cdot \mathrm{ng} / \mathrm{ml}$.

\section{Absorption}

The bioavailability of subcutaneous caplacizumab-yhdp is approximately $90 \%$.

The maximum concentration was observed 6 to 7 hours after subcutaneous dosing of $10 \mathrm{mg}$ caplacizumab-yhdp once daily in healthy subjects.

\section{Distribution}

Caplacizumab-yhdp central volume of distribution is $6.33 \mathrm{~L}$ in patients with aTTP.

\section{Elimination}

The half-life of caplacizumab-yhdp is concentration and target-level dependent.

\section{Metabolism}

The available data suggest target-bound caplacizumab-yhdp is metabolized within the liver. Because caplacizumab-yhdp is a monoclonal antibody fragment, it is expected to be catabolized by various proteolytic enzymes.

\section{Excretion}

The available nonclinical data suggest unbound caplacizumab-yhdp is cleared renally.

\section{Antidrug Antibodies}

No clinically significant differences in the pharmacokinetics of caplacizumab-yhdp were observed in patients with pre-existing or treatment-emergent anti-drug antibodies.

\section{Specific Populations}

No clinically significant differences in the pharmacokinetics of caplacizumab-yhdp were observed based on age (18 to 79 years), sex (66\% females), race (White (83\%) and Black 
$(17 \%))$, blood group $(\mathrm{O}(41 \%)$ and other groups (59\%)), or renal impairment (mild [CrCl: 60 to $90 \mathrm{~mL} / \mathrm{min}$ ], moderate [CrCl: 30 to $60 \mathrm{~mL} / \mathrm{min}$ ] or severe [CrCl: 15 to $30 \mathrm{~mL} / \mathrm{min}$ ]). The effect of hepatic impairment on the pharmacokinetics of caplacizumab-yhdp is unknown [see Use in Specific Populations (8.6)].

\section{Drug Interaction Studies}

No dedicated drug-drug interaction studies with caplacizumab-yhdp have been conducted.

\section{NONCLINICAL TOXICOLOGY}

\subsection{Carcinogenesis, Mutagenesis, Impairment of Fertility}

No studies have been performed to evaluate the potential of caplacizumab-yhdp for carcinogenicity or genotoxicity.

Animal reproduction studies assessing the effects of caplacizumab-yhdp on male and female fertility have not been conducted.

\section{CLINICAL STUDIES}

The efficacy of CABLIVI for the treatment of adult patients with acquired thrombotic thrombocytopenic purpura (aTTP) in combination with plasma exchange and immunosuppressive therapy was established in a pivotal multicenter, randomized, double-blind, placebo-controlled trial (HERCULES) (NCT02553317).

A total of 145 patients were enrolled in the HERCULES study; the median age was 45 (range: 18 to 79 ) years, $69 \%$ were female, $73 \%$ were White. Patients were randomized to either CABLIVI $(n=72)$ or placebo $(n=73)$. Patients in both groups received plasma exchange and immunosuppressive therapy. Patients were stratified according the severity of neurological involvement (Glasgow Coma Scale score $\leq 12$ or 13 to 15 ). Patients with sepsis, infection with $E$. coli 0157, atypical hemolytic uremic syndrome, disseminated intravascular coagulation or congenital thrombotic thrombocytopenic purpura were not eligible for enrollment.

Patients received a single $11 \mathrm{mg}$ CABLIVI bolus intravenous injection or placebo prior to the first plasma exchange on study, followed by a daily subcutaneous injection of $11 \mathrm{mg}$ CABLIVI or placebo after completion of plasma exchange, for the duration of the daily plasma exchange period and for 30 days thereafter. If after the initial treatment course, sign(s) of persistent underlying disease such as suppressed ADAMTS13 activity levels remained present, treatment was extended for 7 day intervals for a maximum of 28 days.

The median treatment duration with CABLIVI was 35 days.

The clinical trial protocol specified the CABLIVI dose as $10 \mathrm{mg}$, to be delivered by withdrawing all of the reconstituted solution from the vial and administering the full amount. A dose recovery study showed that the mean dose that can be withdrawn from a vial is $11 \mathrm{mg}$. Therefore, based on the dose recovery study, the mean dose delivered in the trial was $11 \mathrm{mg}$.

The efficacy of CABLIVI in patients with aTTP was established based on time to platelet count response (platelet count $\geq 150,000 / \mu \mathrm{L}$ followed by cessation of daily plasma exchange within 5 days). Time to platelet count response was shorter among patients treated with CABLIVI, compared to placebo. 
Figure 1: Platelet Response over Time

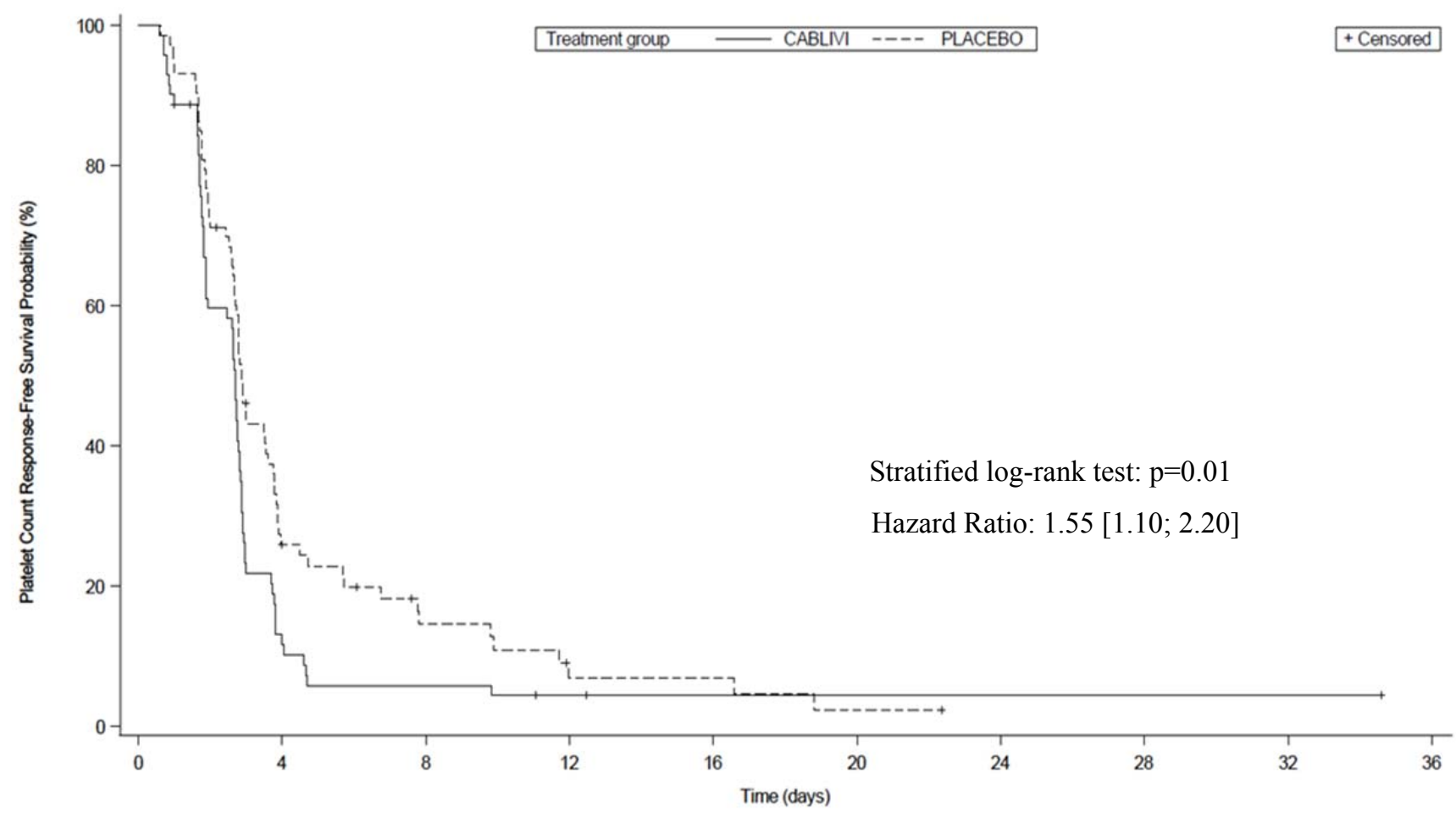

Number of subjects at risk

Treatment with CABLIVI resulted in a lower number of patients with TTP-related death, recurrence of TTP, or at least one treatment-emergent major thromboembolic event (a composite endpoint) during the treatment period (see Table 2).

Table 2: Patients in the HERCULES Study with aTTP-Related Death, a Recurrence of aTTP, or at Least One Treatment-Emergent Major Thromboembolic Event During Study Drug Treatment Period (ITT population)

\begin{tabular}{|l|c|c|}
\hline \multirow{2}{*}{ Number of patients with } & $\begin{array}{c}\text { CABLIVI } \\
\mathbf{N = 7 2}\end{array}$ & $\begin{array}{c}\text { Placebo } \\
\mathbf{N = 7 3}\end{array}$ \\
\cline { 2 - 3 } & $\mathbf{n}(\mathbf{\%})^{*}$ & $\mathbf{n}(\mathbf{\%})$ \\
\hline TTP-related death & 0 & $3(4.1)$ \\
\hline Recurrence of TTP (exacerbation) & $3(4.2)$ & $28(38.4)$ \\
\hline $\begin{array}{l}\text { At least one treatment-emergent } \\
\text { major thromboembolic event }\end{array}$ & $6(8.5)$ & $6(8.2)$ \\
\hline Total $^{\ddagger}$ & $\mathbf{9 ( 1 2 . 7 )}$ & $\mathbf{3 6}(\mathbf{4 9 . 3 )}$ \\
\hline
\end{tabular}

$\mathrm{N}=$ number of patients within the population of interest (by treatment group); $\mathrm{n}=$ number of patients with events; $\mathrm{TTP}=$ thrombotic thrombocytopenic purpura; ITT $=$ intent to treat; * based on 71 patients who received at least one dose of study drug.

$\dagger \quad$ Exacerbation defined as thrombocytopenia after initial recovery of platelet count (platelet count $\geq 150,000 / \mu \mathrm{L}$ with subsequent stop of daily plasma exchange within 5 days) that required reinitiation of daily plasma exchange 
during the 30-day post daily plasma exchange period.

$\mathrm{p}<0.0001$

The proportion of patients with a recurrence of TTP in the overall study period (the drug treatment period plus the 28-day follow-up period after discontinuation of drug treatment) was lower in the CABLIVI group (9/72 patients [13\%]) compared to the placebo group (28/73 patients $[38 \%](\mathrm{p}<0.001)$. In the 6 patients in the CABLIVI group who experienced a recurrence of TTP during the follow-up period (i.e., a relapse defined as recurrent thrombocytopenia after initial recovery of platelet count (platelet count $\geq 150,000 / \mu \mathrm{L}$ ) that required reinitiation of daily plasma exchange, occurring after the 30-day post daily plasma exchange period), ADAMTS13 activity levels were $<10 \%$ at the end of the study drug treatment, indicating that the underlying immunological disease was still active at the time CABLIVI was stopped.

\section{HOW SUPPLIED/STORAGE AND HANDLING}

\subsection{How Supplied}

CABLIVI (caplacizumab-yhdp) for injection is a sterile, white, preservative-free, lyophilized powder in a single-dose vial. Each carton (NDC 58468-0225-1) contains:

- one 11 mg CABLIVI single-dose vial (NDC 58468-0227-1)

- one $1 \mathrm{~mL}$ Sterile Water for Injection, USP, prefilled glass syringe (diluent for CABLIVI) (NDC 58468-0229-1)

- one sterile vial adapter

- one sterile hypodermic needle (30 gauge)

- two individually packaged alcohol swabs

\subsection{Storage}

Store refrigerated at $2^{\circ} \mathrm{C}$ to $8^{\circ} \mathrm{C}\left(36^{\circ} \mathrm{F}\right.$ to $\left.46^{\circ} \mathrm{F}\right)$ in the original carton to protect from light. Do not freeze. Unopened vials may be stored in the original carton at room temperature up to $30^{\circ} \mathrm{C}$ $\left(86^{\circ} \mathrm{F}\right)$ for a single period of up to 2 months. Do not return CABLIVI to the refrigerator after it has been stored at room temperature.

\section{PATIENT COUNSELING INFORMATION}

Advise the patient to read the FDA-approved patient labeling (Instructions for Use).

Bleeding [see Warnings and Precautions (5.1)]

- Advise patients that bruising and bleeding may occur more easily, that nose bleeds and bleeding of gums may occur, and that it may take them longer than usual to stop bleeding. Advise patients to contact their healthcare provider immediately if excessive bleeding or bruising occur.

- Advise patients to inform their healthcare provider before scheduling any elective surgery, dental procedure or other invasive interventions.

Manufactured by:

Ablynx N.V.

Technologiepark 21 
9052 Ghent (Zwijnaarde), Belgium

U.S. License No. 2085

Distributed by:

Genzyme Corporation

Cambridge, MA 02142

CABLIVI is a registered trademark of Ablynx N.V., Belgium (C)2018 Ablynx NV. All rights reserved. 


\section{Instructions for Use \\ CABLIVI ${ }^{\circledR}$ (cab-LIV-ee) \\ (caplacizumab-yhdp) \\ for injection \\ Single-dose vial}

Be sure that you read, understand, and follow this Instructions for Use before you inject CABLIVI and each time you get a refill. There may be new information. Your healthcare provider should show you how to prepare and inject CABLIVI properly before your first injection. Talk to your healthcare provider if you have any questions.

\section{I mportant information:}

- Make sure that the name CABLIVI appears on the carton and vial label.

- For each injection, one CABLIVI carton is needed. Only use the vial one time.

- Only use the supplies that are provided in the carton to prepare your prescribed dose.

- Do not use CABLI VI after the expiration date on the carton.

- Do not reuse any of the supplies. After your injection, throw away (dispose of) the used vial with any remaining CABLIVI liquid in it. Throw away (dispose of) the used vial with the adapter attached and the syringe with the needle attached in a FDA-cleared sharps disposal container. See "Step 13: Throw away (dispose of) the used syringe and vial" at the end of this Instructions for Use for more disposal information.

\section{How should I store CABLIVI?}

- Store CABLIVI in the refrigerator between $36^{\circ} \mathrm{F}$ to $46^{\circ} \mathrm{F}\left(2^{\circ} \mathrm{C}\right.$ to $\left.8^{\circ} \mathrm{C}\right)$.

- Use the mixed CABLIVI solution immediately. The mixed CABLIVI solution can be stored for up to 4 hours in the refrigerator at $36^{\circ} \mathrm{F}$ to $46^{\circ} \mathrm{F}\left(2^{\circ} \mathrm{C}\right.$ to $\left.8^{\circ} \mathrm{C}\right)$.

- If needed, unopened CABLIVI vials may be stored at room temperature (up to $30^{\circ} \mathrm{C}$ or $86^{\circ} \mathrm{F}$ ) in the original carton for a single period of up to two months. Write the date removed from the refrigerator in the space provided on the carton.

- Do not return CABLI VI to the refrigerator after it has been stored at room temperature.

- Do not freeze CABLIVI.

- Keep CABLIVI in the carton that it came in to protect it from light.

- Keep CABLI VI and all medicines out of the reach of children.

\section{Each CABLI VI carton contains:}

- 1 vial of CABLIVI

- 1 prefilled syringe containing $1 \mathrm{~mL}$ Sterile Water for I njection, USP (diluent for CABLIVI)

- 1 sterile vial adapter

- 1 sterile needle

- 2 alcohol swabs

- Additional supplies needed:

o FDA-cleared sharps disposal container. See "Step 13: Throw away (dispose of) the used syringe" at the end of this Instructions for Use for more disposal information.

o Cotton balls 


\section{Contents of CABLIVI carton}

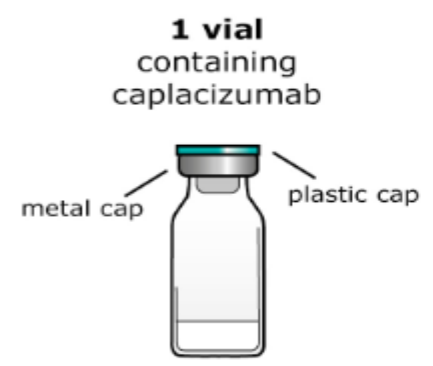

1 sterile vial adapter

vial adapter package

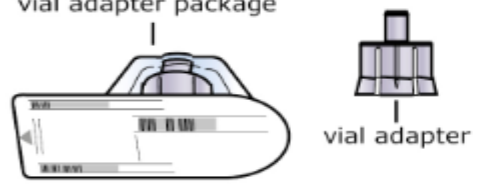

2 alcohol swabs

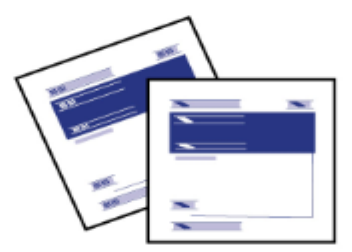

1 sterile needle

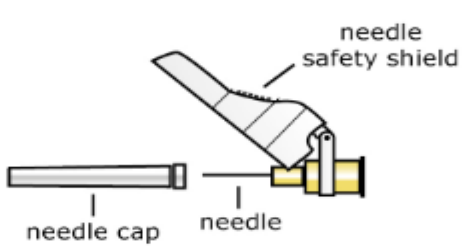

1 prefilled syringe containing $1 \mathrm{~mL}$ Sterile Water for Injection, USP

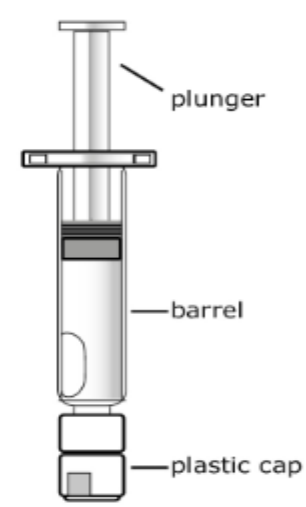

\section{Additional supplies needed (not in CABLIVI carton)}

Cotton balls

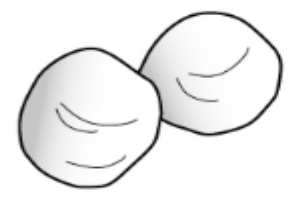

Sharps disposal container

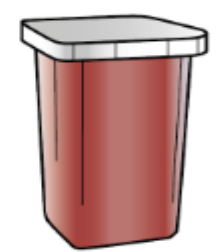

\section{Before preparing a dose of CABLIVI:}

- Wash your hands well with soap and water.

- Prepare a clean flat surface.

- Check to make sure the carton contains all of the items needed to prepare a dose.

- Check the expiration date (See Figure A). Do not use CABLIVI if the date has passed.

- Do not use CABLIVI if the packaging or any supplies inside of the carton are damaged in any way.

\section{Step 1: Bring the vial and syringe to room} temperature

- Place all the supplies in the carton on the clean flat surface.

- If the carton was not stored at room temperature, allow the vial and the syringe to reach room temperature by holding them in your hands for 10 seconds ( See Figure B). Do not use any other way to warm up the vial and syringe.

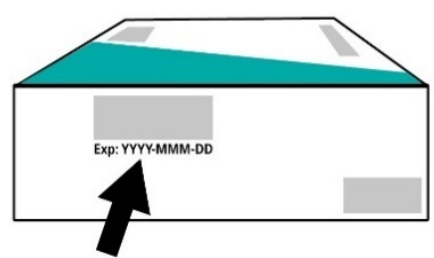

Figure A

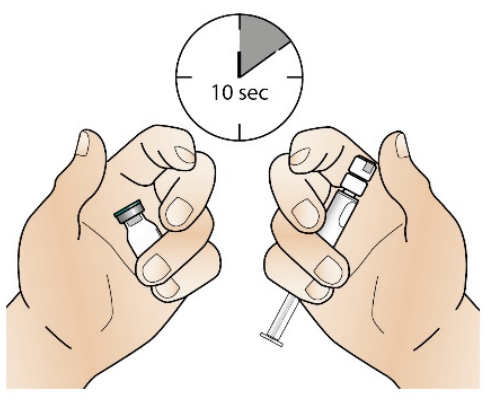

Figure B 


\section{Step 2: Clean the rubber stopper}

- Remove the green plastic flip-off cap from the metal cap of the vial (See Figure C). Do not use the vial if the green plastic cap is missing.

- Clean the exposed rubber stopper using one alcohol pad to wipe it and allow it to dry for a few seconds (See Figure D).

- After cleaning the rubber stopper, do not touch it or allow it to touch any surface.

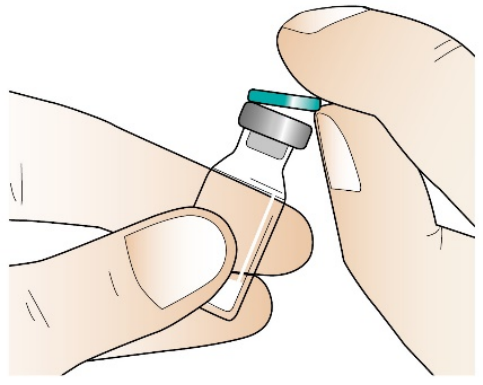

Figure C

\section{Step 3: Attach the vial adapter}

- Take the vial adapter and remove the paper cover (See Figure E). Leave the vial adapter in its opened plastic packaging for now. Do not touch the adapter itself.

- Place the adapter over the vial, while keeping the adapter in its plastic packaging.

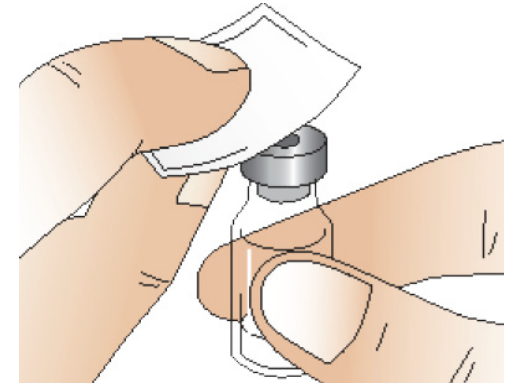

Figure D

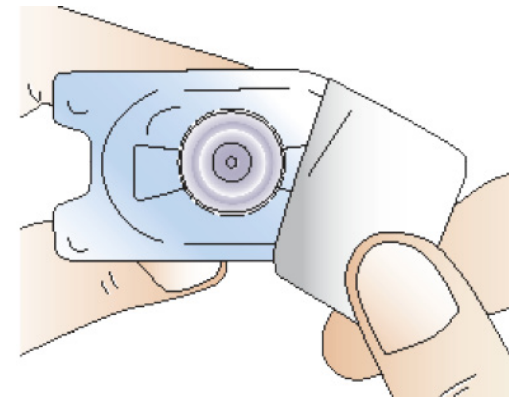

Figure E

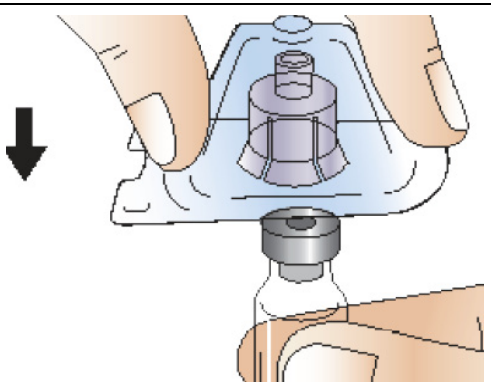

Figure F

\section{Step 4: Prepare the syringe}

- Pick up the syringe.

- While holding the syringe with one hand, break off the white plastic cap by snapping at the perforation of the cap with your other hand (See Figure G).

- Do not use the syringe if the white plastic cap is missing, loose, or damaged.

- Do not touch the syringe tip or allow it to come into contact with any surfaces.

- Lay the syringe on the clean flat surface.

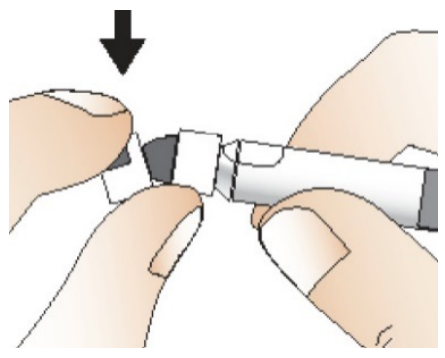

Figure G 


\section{Step 5: Connect the syringe to adapter and vial}

- Remove the plastic packaging from the adapter attached to the vial by holding the vial with one hand, pressing the sides of the adapter packaging with your other hand, and then lifting the packaging upwards (See Figure H).

- Be sure that the adapter does not detach from the vial.

- Hold the adapter with the attached vial with one hand. Place the tip of the syringe on the connector part of the vial adapter using the other hand.

- Gently lock the syringe into the vial adapter by turning it clockwise until it cannot twist any further (See Figure I).

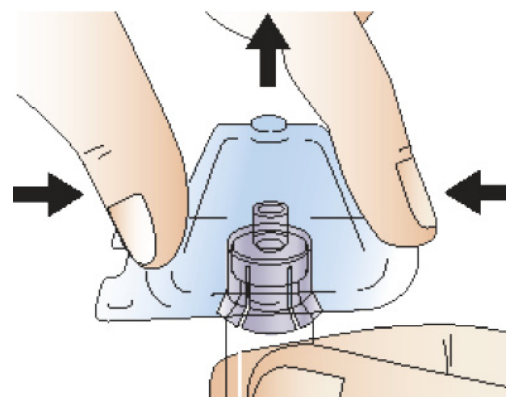

Figure H

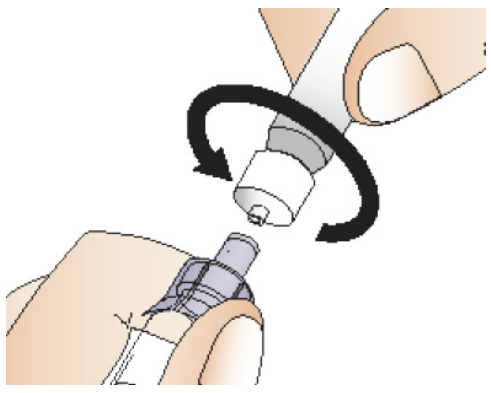

Figure I

\section{Step 6: Prepare the solution}

- Place the vial upright on the flat surface with the syringe pointing downwards.

- Slowly push the syringe plunger down until the syringe is empty (See Figure J ). Do not remove the syringe from the vial adapter.

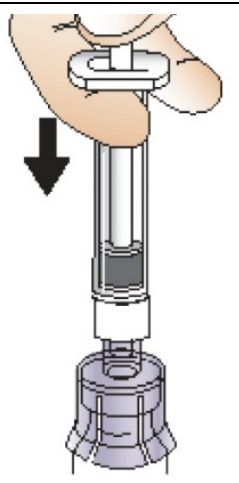

Figure J

- With the syringe still connected to the vial adapter, gently swirl the vial, with syringe attached, until the powder is dissolved in the vial (See Figure $K)$. Do not shake the vial.

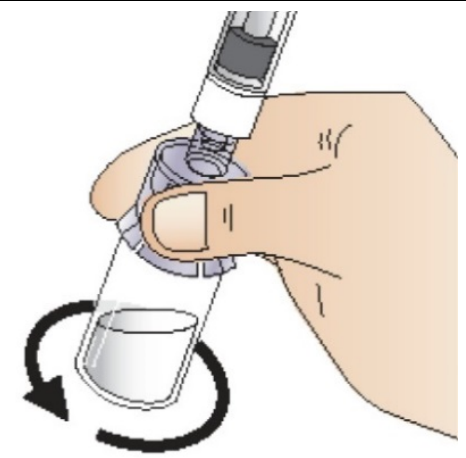

Figure K 
- Allow the vial with the attached syringe to stand on the flat surface for $\mathbf{2}$ minutes at room temperature to allow the powder to completely dissolve (See Figure L). The plunger may rise up by itself again, this is normal.

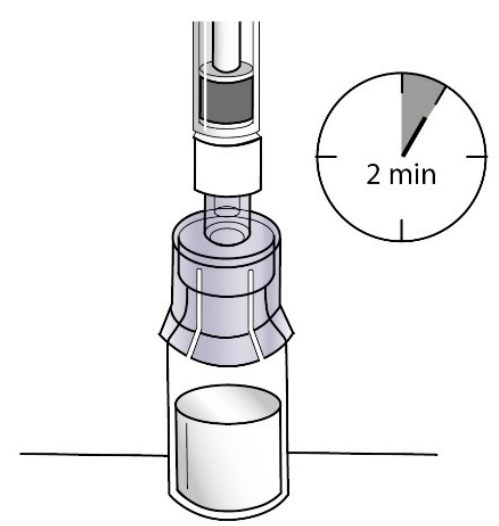

Figure L

\section{Step 7: Draw up solution}

- Check the solution in the vial for particles, cloudiness, or clumps. All powder must be fully dissolved and the solution must be clear. Do not use the medicine if you see particles, cloudiness, or clumps. Use a new carton of CABLIVI or call your healthcare provider.

- Slowly press the syringe plunger fully down.

- Keep the syringe on the vial and turn the vial, adapter and syringe upside down.

- Slowly pull the plunger down to withdraw all of the solution from the vial into the syringe (See Figure M). Do not shake it.

\section{Step 8: Detach the syringe}

- After drawing up the solution into the syringe, turn the vial, adapter and syringe back to the starting position and place on the flat surface (See Figure N).

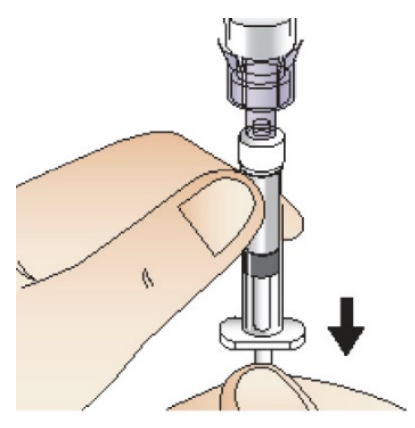

Figure $M$

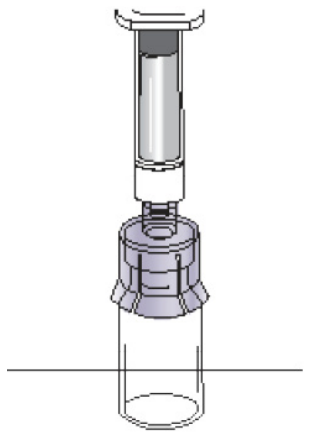

Figure $\mathbf{N}$

- Detach the filled syringe from the adapter by holding the vial and adapter in one hand and gently twisting the syringe counter-clockwise with the other hand (See Figure $\mathbf{0}$ ).

- Throw away the vial and the attached adapter into a sharps disposal container.

- Do not touch the syringe tip or allow it to touch the clean flat surface. Place the syringe on the clean flat surface.

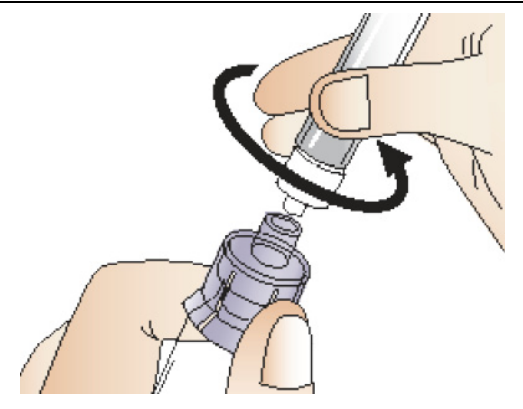

Figure 0 


\section{Step 9: Attach the needle}

- Open the needle package by using both thumbs to pull apart the packaging (See Figure P).

- Remove the needle from the package.

- Attach the needle with the needle cap to the syringe by turning clockwise until it cannot twist any further (See Figure $\mathbf{Q}$ ). Do not remove the needle cap.

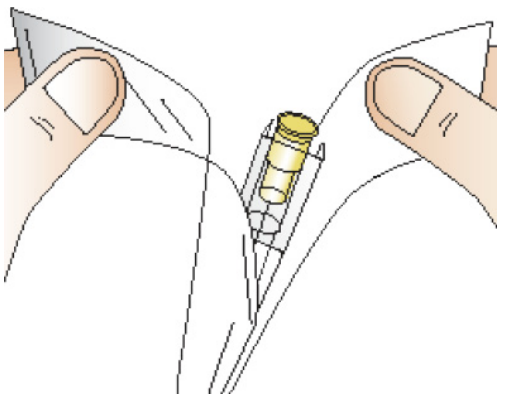

Figure $\mathbf{P}$

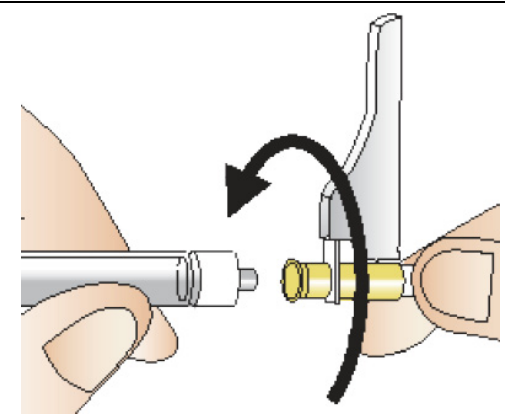

Figure Q

- Pull back the needle safety shield (See Figure R).

\section{Step 10: Prepare your injection site}

- Select an injection site on your stomach

(abdomen) (See Figure S). Avoid the 2-inch area around your belly button (navel). It is important to avoid injecting in the same site on your abdomen that you used for a previous injection on consecutive days.

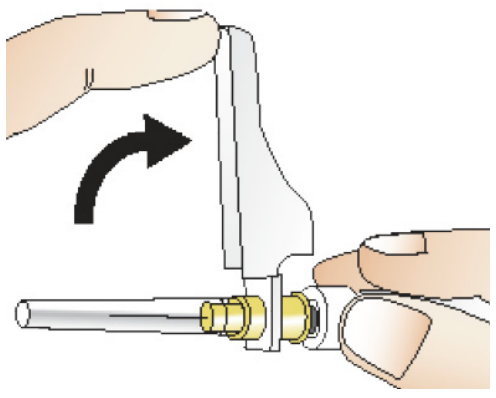

Figure $\mathbf{R}$

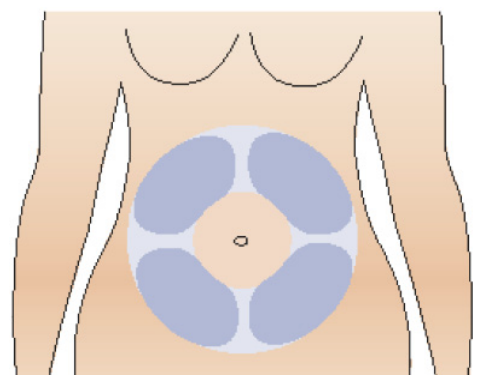

Figure S

- Clean your injection site with an alcohol pad (See Figure T). Let your skin dry.

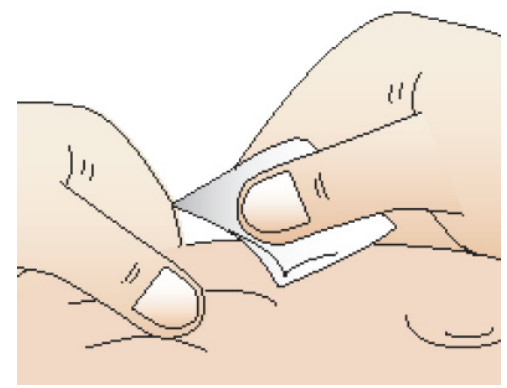

Figure T 


\section{Step 11: Giving your injection}

- Carefully remove the needle cap from the needle and throw it away in a sharps disposal container (See Figure $\mathbf{U}$ ). Make sure the needle does not touch anything before the injection.

- Hold the syringe at eye level with the needle pointing upwards.

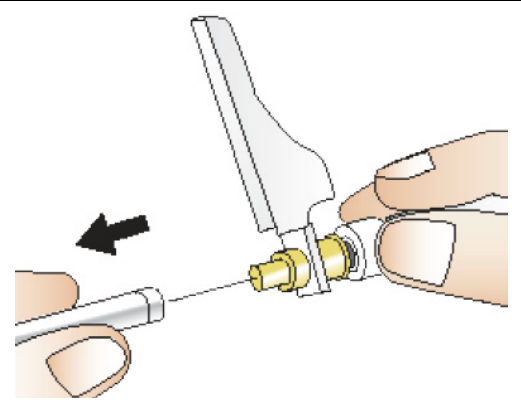

Figure U

- Check to see if there are any air bubbles. If there are any air bubbles, remove them by tapping the side of the syringe with your finger until they rise towards the tip (See Figure V).

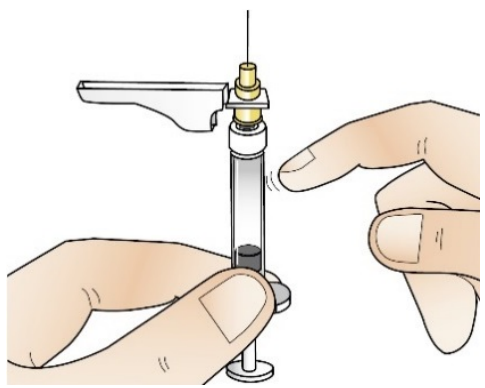

Figure V

- Then, slowly push the plunger up until a small amount of liquid drips from the needle (See Figure W).

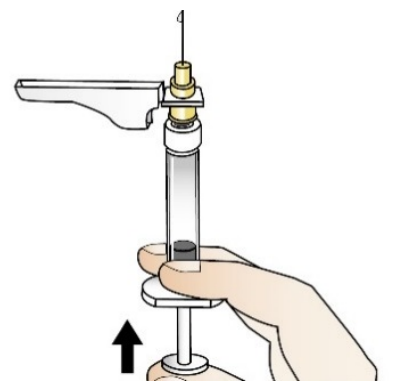

Figure $\mathbf{W}$

- Gently use one hand to pinch the skin that has been cleaned between your thumb and forefinger, making a fold (See Figure $\mathbf{X}$ ).

- Hold the pinch during the entire injection.

- Use your other hand to insert the needle all the way into your skin fold at a 45 to 90 -degree angle (See Figure $\mathbf{Y}$ ).

- Push down on the plunger of the syringe until all of the solution is injected into your skin.

- Pull out the needle at the same angle you inserted it. Do not rub your injection site.

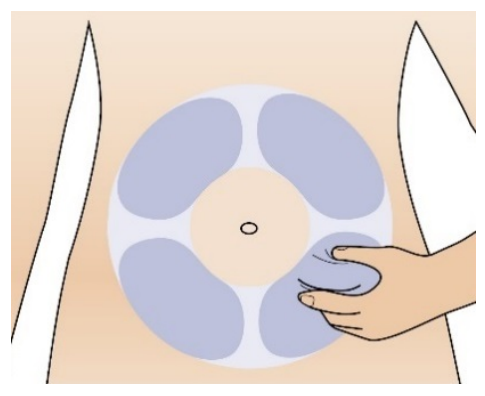

Figure $X$

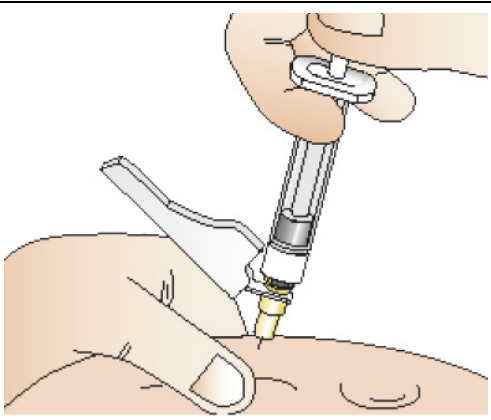

Figure $Y$ 


\section{Step 12: After your injection}

- Right after your injection, move the needle safety shield over the needle until it clicks into place to activate the shield (See Figure $\mathbf{Z}$ ).

- In case you are bleeding at the injection site, place a cotton ball over the skin right away. Press gently on the cotton ball until the bleeding has stopped. If bleeding does not stop, call your healthcare provider.

- If your injection site becomes red or sore, call your healthcare provider right away.

\section{Step 13: Throw away (dispose of) the used syringe} and vial.

- Throw away the syringe with the needle and the vial with the adapter in a FDA-cleared sharps disposal container right away after use. Do not throw away (dispose of) loose needles and syringes with your household trash.

- If you do not have a FDA-cleared sharps disposal container, you may use a household container that is:

o made of a heavy-duty plastic,

o can be closed with a tight-fitting, punctureresistant lid, without sharps being able to come out,

o upright and stable during use,

o leak-resistant, and

o properly labeled to warn of hazardous waste inside the container.

- When your sharps disposal container is almost full, you will need to follow your community guidelines for the right way to dispose of your sharps disposal container. There may be state or local laws about how you should throw away used needles and syringes. For more information about safe sharps disposal, and for specific information about sharps disposal in the state in which you live, go to the FDA's website at:

http://www.fda.gov/safesharpsdisposal.

- Do not dispose of your used sharps disposal container in your household trash unless your community guidelines permit this. Do not recycle your used sharps disposal container.

This Instructions for Use has been approved by the U.S. Food and Drug Administration.

Manufactured by:

Ablynx N.V.

Ghent (Zwijnaarde), Belgium

U.S. License No. 2085

Distributed by:

Genzyme Corporation 
Cambridge, MA 02142

Issued: February 2019 